\title{
Predictive clinical factors in rheumatoid arthritis using disease activity and functional score
}

\author{
Worakan Tipsing ${ }^{1}$, Kittisak Sawanyawisuth² ID \\ ${ }^{1}$ Srinakharinwirot University, Bangkok, Thailand \\ ${ }^{2}$ Khon Kaen University, Khon Kaen, Thailand
}

\begin{abstract}
Objectives: Rheumatoid arthritis (RA) is a chronic inflammatory arthritis that may lead to severe joint pain. There are several scores to evaluate the disease activity of RA. This study aimed to evaluate if clinical factors which representing activity scores and health assessment score.

Material and methods: This study was conducted prospectively by including adult patients with RA. Clinical factors and 5 RA disease activity and health assessment scores were evaluated. Each activity score was executed for clinical predictors by using multivariate linear regression analysis. Results: There were 33 female adult patients in the study. The average (SD) age was 52.33 (11.11) years, while the duration of RA was 7.65 years. The DAS28 ESR had 1 predictor: RA duration with a coefficient of -0.04 . For DAS28 CRP, CDAl, and SDAI scores, body mass index (BMI) and RA duration were independent factors for the scores with negative coefficient values. For the HAQ score, both age and rheumatoid duration were positively associated with the score. The coefficients of both factors were 0.02 and 0.03 , respectively.

Conclusions: Age, RA duration, and BMI were associated with RA activity and functional score. Body mass index is a potential modifiable factor that may be associated with RA activities.
\end{abstract}

Key words: rheumatoid arthritis, clinical factors predictive, functional score, quality of life.

\section{Introduction}

Rheumatoid arthritis (RA) is a chronic inflammatory arthritis that may lead to severe joint pain, limitation of daily activities, and long-term disabilities. Treatments are not only aimed at reducing joint inflammation/pain but also at preventing further joint destruction and providing better functional health status [1]. There are several methods to evaluate pain indicating disease activity and functional status, including the disease activity score (DAS), simplified disease activity index (SDAI), clinical disease activity index (CDAI), and Health Assessment Questionnaire (HAQ) [2].

These scores are widely used and can be calculated online. The main advantage of these scores is to assist clinicians in deciding the RA treatments for both clinical trials and clinical practices. For example, a cut-off point of 15 for the SDAl indicated moderate to high RA activity with sensitivity of $90 \%$ and specificity of $86 \%$ [2].
Even though the scores are useful in RA evaluation, it is limited whether these scores are influenced by patient characteristics or clinical factors. Some clinical factors may be modifiable, such as body mass index (BMI) or lipid profiles. If modifiable clinical factors have an influence on RA activity scores, they may be used as additional tools to control RA.

\section{Material and methods}

This study was conducted prospectively at Panyananthaphikkhu Chonprathan Medical Center (PCMC). The inclusion criteria were adult patients diagnosed as RA by ACR 2012 criteria without other diseases interfering in tender and oedematous joint count, treated at a rheumatology outpatient clinic, and willing to participate the study. Exclusion criteria were those with other autoimmune diseases, or serious co-morbid diseases such as cancer and severe infection. This study was a part of 
Table I. Baseline characteristics of rheumatoid arthritis patients evaluated for disease activity $(n=33)$

\begin{tabular}{|lc|}
\hline Factors & $\begin{array}{c}\text { Number (percentage) } \\
\text { or mean (SD) }\end{array}$ \\
\hline Age, years & $52.33(11.11)$ \\
\hline RA duration [years] & $7.65(9.08)$ \\
\hline RF & $16(48.48)$ \\
\hline ACPA level [IU/ml] & $380.48(733.78)$ \\
\hline Numbers of DMARDs use & $2.87(1.02)$ \\
\hline Steroid dose [mg/day] & $2.80(3.68)$ \\
\hline Diabetes mellitus & $4(12.12)$ \\
\hline Hypertension & $15(45.45)$ \\
\hline Dyslipidaemia & $17(51.52)$ \\
\hline $\begin{array}{l}\text { Iron deficiency anaemia } \\
\text { or thalassaemia }\end{array}$ & $3(9.09)$ \\
\hline Asthma & $1(3.03)$ \\
\hline BMI [kg/m²] & $23.74(4.67)$ \\
\hline
\end{tabular}

ACPA - anti-citrullinated protein antibodies, BMI - body mass index, DMARDs - disease-modifying antirheumatic drugs, $R A$ - rheumatoid arthritis, RF-rheumatoid factor.

Table II. Disease activity and functional status by various scores in rheumatoid arthritis patients $(n=33)$

\begin{tabular}{|lc|}
\hline Scores & Median $\left(1^{\text {st }}-3^{\text {rd }}\right.$ quartile range $)$ \\
\hline DAS28 ESR & $3.31(3.00-4.29)$ \\
\hline DAS28 CRP & $2.45(1.88-3.15)$ \\
\hline CDAI & $6(4-12)$ \\
\hline SDAI & $6.39(4.60-14.00)$ \\
\hline HAQ & $0.25(0.13-1.00)$ \\
\hline
\end{tabular}

$C D A I$ - Clinical disease activity index, $C R P-C$-reactive protein, $D A S$ - Disease activity score, ESR - erythrocyte sedimentation rate, HAQ - Health Assessment Questionnaire, SDAI - Simplified disease activity index.

the clinical study of RA. The study period was between November 2016 and April 2019.

The study protocol complied with the Declaration of Helsinki. All participants signed an Informed Consent Form, which was previously approved by the institutional review board of ethical human research (ID no. 7/2559).

The eligible patients were evaluated baseline characteristics including age, gender, and co-morbid disease, and factors-related to RA including disease duration, treatment modalities, and laboratory results. The 5 disease activity and health assessment scores were also reported including DAS28 erythrocyte sedimentation rate or DAS28 ESR, DAS28 C-reactive protein or DAS28 CRP, CDAI, SDAI, and HAQ [2].

\section{Statistical analyses}

Baseline characteristics of eligible patients were calculated by descriptive statistics. Each activity score was executed for clinical predictors by using multivariate linear regression analysis. A univariate linear regression was initially evaluated for the coefficient. Subsequent stepwise multivariate linear regression was performed. Factors with the $p$-value of 0.2 or less by the univariate analysis were included in the model, while the factors with $p$-value of over 0.25 were removed from the final model. Significant factors by the final model predictive for the scores were defined by the $p$-value of less than 0.05 . Coefficient, standard error, and $p$-values of each factor in the final model were reported.

The statistical analyses were performed using STATA software, version 10.1 (College Station, Texas, USA).

\section{Results}

There were 33 female adult patients who met the study criteria. The average (SD) age was 52.33 (11.11) years, while the duration of RA was 7.65 years (Table I). Rheumatoid factor (RF) was positive in 16 patients (48.48\%) with the SD number of DMARDs use of 2.87 items. Dyslipidaemia was the most common co-morbid disease (51.52\%). The average BMI was $23.74 \mathrm{~kg} / \mathrm{m}^{2}$.

The median disease activity scores were in the low disease activity range for DAS28 CRP, CDAI, and SDAI, while the DAS28 ESR score was in the moderate range (3.31), as shown in Table II. Factors remaining in the final model for the 5 scores are shown in Table III.

The final model for DAS28 CRP and SDAI had the maximum remaining factors of 6 . Only the DAS28 ESR had 1 independent factor: RA duration with a coefficient of -0.04 , while the other scores had 2 independent factors. For DAS28 CRP, CDAI, and SDAI scores, the 2 independent factors were negatively associated with the scores. The highest coefficient was -6.71 in the correlation between RF and SDAI ( $p$-value 0.04). For the HAQ score, both age and RA duration were positively associated with the score. The coefficients of both factors were 0.02 and 0.03 , respectively.

\section{Discussion}

To date, several reports have found an association between BMI and disease activity, particularly for DAS28 [3-9]. However, data are still conflicting. A meta-analysis from 4 studies found that DAS28 was significantly higher or more severe if the BMI was over $30 \mathrm{~kg} / \mathrm{m}^{2}$, compared to those with lower BMI. The mean difference of both groups was $+0.14 ; p=0.04$ [4]. Another study found that those with BMI over 25 had lower DAS28 
Table III. Factors associated with various disease activity scores by multivariate linear regression analyses in rheumatoid arthritis patients $(n=33)$

\begin{tabular}{|lcccccccccccccccc|}
\hline Parameters & \multicolumn{2}{c}{ DAS28 ESR } & \multicolumn{3}{c}{ DAS28 CRP } & \multicolumn{4}{c}{ CDAI } & \multicolumn{4}{c}{ SDAI } & \multicolumn{3}{c|}{ HAQ } \\
\hline & Coef. & SE & $p$ & Coef. & SE & $p$ & Coef. & SE & $p$ & Coef. & SE & $p$ & Coef. & SE & $p$ \\
\hline Age & & & & & & & -0.15 & 0.10 & 0.13 & -0.14 & 0.11 & 0.22 & 0.02 & 0.01 & 0.04 \\
\hline RA duration & -0.04 & 0.02 & 0.03 & -0.03 & 0.02 & 0.14 & & & & -0.21 & 0.15 & 0.18 & 0.03 & 0.01 & 0.02 \\
\hline RF & & & & -0.81 & 0.36 & 0.03 & -5.59 & 2.61 & 0.04 & -6.71 & 3.19 & 0.04 & -0.38 & 0.24 & 0.12 \\
\hline ACPA & 0.00 & 0.00 & 0.25 & 0.00 & 0.00 & 0.14 & & & & 0.00 & 0.00 & 0.09 & & \\
\hline DMARDs & 0.19 & 0.16 & 0.24 & 0.21 & 0.14 & 0.15 & & & & & & & & & \\
\hline Steroid & 0.08 & 0.05 & 0.09 & 0.07 & 0.04 & 0.07 & 0.54 & 0.30 & 0.08 & 0.42 & 0.34 & 0.22 & 0.04 & 0.02 & 0.17 \\
\hline Dyslipidaemia & & & & & & & & & & & & & -0.44 & 0.24 & 0.08 \\
\hline BMI & & & & -0.08 & 0.03 & 0.03 & -0.70 & 0.25 & 0.14 & -0.83 & 0.29 & 0.01 & & & \\
\hline
\end{tabular}

ACPA - anti-citrullinated protein antibodies, BMI - body mass index, CDAl-Clinical disease activity index, DAS - Disease activity SCore, DMARDs - disease-modifying antirheumatic drugs, HAQ - Health Assessment Questionnaire, SDAl - Simplified disease activity index, $R A$ - rheumatoid arthritis, RF-rheumatoid factor.

or less severe disease than those with lower BMI [5] The average DAS28 of both groups were 5.05 vs. 5.57; $p=0.016$. Note that the latter study was analysed by using descriptive statistics, which may be interfered by confounding factors.

This study found that BMI is negatively associated with the DAS28 CRP, CDAI, and SDAI scores. These data indicated that low BMI patients may have more severe disease. The strength of our analysis is that it was adjusted for other factors or controlled for other confounding factors. The low BMI was shown to be related with more bone erosion (adjusted beta of -0.448), as shown by several other reports [6-9]. A report found that the disease activity scores were related more to radiographic changes [10] resulting in negative association of BMI to DAS28 CRP, CDAI, and SDAI (Table III).

Another factor negatively associated with DAS28 CRP, CDAl, and SDAI was positive RF (Table III). These data are compatible with a previous study from Korea [11]. The seronegative group had higher DAS than those with a seropositive group (DAS28 6.1 vs. 4.7; $p=0.006$ ). This study also showed that longer disease duration after treatment resulted in lower disease activity regardless of serology test. These results were similar to those in the current study (Table III), which showed that RA duration was significantly lower disease activity (DAS28 ESR) with a coefficient of -0.04 .

For $\mathrm{HAQ}$, it has been known that HAQ score increases with age, indicating greater disability [2]. After adjusting for age and other variables, RA duration was positively associated with the HAQ score. Two previous studies support that longer RA duration is associated with poorer functional status $[12,13]$.

Another study found different findings [14]: RF, but not age, was significantly associated with the HAQ score $(r=0.63 ; p$-value 0.0043). However, there was no adjustment with other factors in that study. The final model in our study was adjusted for other confounding factors and found no significant relation between $\mathrm{HAQ}$ and RF (Table III).

There are some limitations to this study: only a small group of female patients took part; patients lacked complex comorbid diseases such as liver disease, renal disease, stroke, or CAD; and few patients had high disease activity.

\section{Conclusions}

Age, RA duration, and BMI were associated with RA activity and functional score. BMI is a potential modifiable factor that may be associated with RA activities.

The authors declare no conflict of interest.

\section{References}

1. Smolen JS, Breedveld FC, Burmester GR, et al. Treating rheumatoid arthritis to target: 2014 update of the recommendations of an international task force. Ann Rheum Dis 2016; 75 : 3-15, DOI: 10.1136/annrheumdis-2015-207524.

2. Aletaha D, Smolen JS. The definition and measurement of disease modification in inflammatory rheumatic diseases. Rheum Dis Clin North Am 2006; 32: 9-44, DOI: 10.1016/j. rdc.2005.09.005.

3. Di Carlo M, Salaffi F, Gremese E, et al. Body mass index as a driver of selection of biologic therapy in rheumatoid arthritis. Results from the US-CLARA study. Eur J Intern Med 2019; 66: 57-61, DOI: 10.1016/j.ejim.2019.05.017.

4. Vidal C, Barnetche T, Morel J, et al. Association of body mass index categories with disease activity and radiographic joint damage in rheumatoid arthritis: a systematic review and meta-analysis. J Rheumatol 2015; 42: 2261-2269, DOI: 10.3899/jrheum.150224. 
5. Gamal SM, Alkemary AK, Abdo MA, El Dakrony AHM. Low body mass index in long standing rheumatoid arthritis: relation to RA disease activity and functional indices. Reumatismo 2018; 70: 72-77, DOI: 10.4081/reumatismo.2018.999.

6. Velpula UD, Agrawal S, Thomas J, et al. Low body mass in dex is adversely associated with radiographic joint damage in Indian patients with early rheumatoid arthritis. J Rheumatol 2011; 38: 434-438, DOI: 10.3899/jrheum.100535.

7. Kaufmann J, Kielstein V, Kilian S, et al. Relation between body mass index and radiological progression in patients with rheumatoid arthritis. J Rheumatol 2003; 30: 2350-2355.

8. van der Helm-van Mil AH, van der Kooij SM, Allaart CF, et al. A high body mass index has a protective effect on the amount of joint destruction in small joints in early rheumatoid arthritis. Ann Rheum Dis 2008; 67: 769-774, DOI: 10.1136/ ard.2007.078832.

9. Westhoff G, Rau R, Zink A. Radiographic joint damage in early rheumatoid arthritis is highly dependent on body mass index. Arthritis Rheum 2007; 56; 3575-3582, DOI: 10.1002/ art.23033.

10. Klarenbeek NB, Koevoets R, van der Heijde DM, et al. Association with joint damage and physical functioning of nine composite indices and the 2011 ACR/EULAR remission criteria in rheumatoid arthritis. Ann Rheum Dis 2011; 70: 1815-1821, DOI: 10.1136/ard.2010.149260.

11. Choi S, Lee KH. Clinical management of seronegative and seropositive rheumatoid arthritis: a comparative study. PLoS One 2018; 13: e0195550, DOI: 10.1371/journal.pone.0195550.

12. Fonseca JE, Canhão H, Teixeira da CJ, et al. Global functional status in rheumatoid arthritis: disease duration and patient age. Clin Rheumatol 2002; 21: 32-34, DOI: 10.1007/ s100670200008.

13. Serbo B, Jajić I. Relationship of the functional status, duration of the disease and pain intensity and some psychological variables in patients with rheumatoid arthritis. Clin Rheumatol 1991; 10: 419-422, DOI: 10.1007/BF02206663.

14. Boyd TA, Bonner A, Thorne C, et al. The relationship between function and disease activity as measured by the HAQ and DAS28 varies over time and by rheumatoid factor status in early inflammatory arthritis (EIA). Results from the $\mathrm{CATCH}$ cohort. Open Rheumatol J 2013; 7: 58-63, DOI: 10.2174/ 1874312901307010058. 